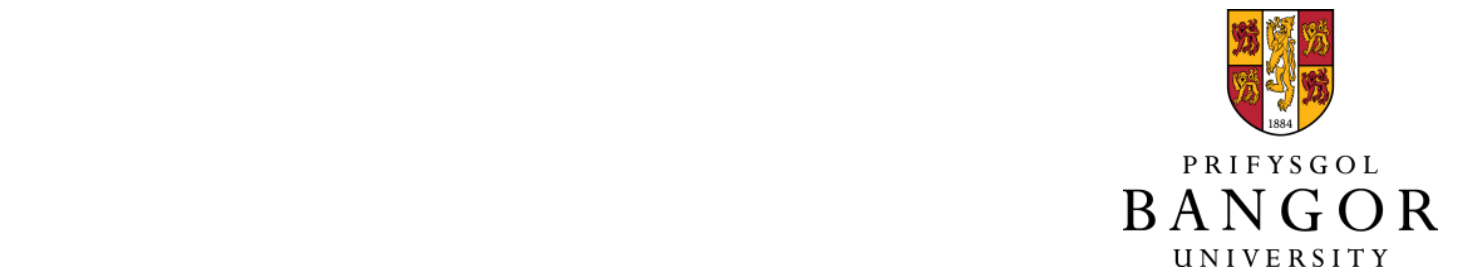

\title{
Experimental Demonstration of a Real-Time Digital Filter Multiple Access PON with Low Complexity DSP-Based Interference Cancellation
}

Al-Rawachy, Ehab; Giddings, Roger Philip; Tang, Jianming

\section{Journal of Lightwave Technology}

DOI:

10.1109/JLT.2019.2923546

Published: 01/09/2019

Peer reviewed version

Cyswllt i'r cyhoeddiad / Link to publication

Dyfyniad o'r fersiwn a gyhoeddwyd / Citation for published version (APA):

Al-Rawachy, E., Giddings, R. P., \& Tang, J. (2019). Experimental Demonstration of a Real-Time Digital Filter Multiple Access PON with Low Complexity DSP-Based Interference Cancellation. Journal of Lightwave Technology, 37(17), 4315-4329. https://doi.org/10.1109/JLT.2019.2923546

Hawliau Cyffredinol / General rights

Copyright and moral rights for the publications made accessible in the public portal are retained by the authors and/or other copyright owners and it is a condition of accessing publications that users recognise and abide by the legal requirements associated with these rights.

- Users may download and print one copy of any publication from the public portal for the purpose of private study or research.

- You may not further distribute the material or use it for any profit-making activity or commercial gain

- You may freely distribute the URL identifying the publication in the public portal ?

Take down policy

If you believe that this document breaches copyright please contact us providing details, and we will remove access to the work immediately and investigate your claim. 


\title{
Experimental Demonstration of a Real-Time Digital Filter Multiple Access PON with Low Complexity DSP-Based Interference Cancellation
}

\author{
Ehab Al-Rawachy, Roger Philip Giddings, Member, IEEE and Jianming Tang, Member, IEEE
}

\begin{abstract}
A 4-channel, upstream, digital filter multiple access (DFMA) passive optical network (PON) is experimentally demonstrated for the first time in a $26 \mathrm{~km}$ SMF IMDD system employing real-time ONUs and OLT, which are dynamically reconfigurable in terms of channel spectral allocation and signal modulation parameters. To combat physical channel frequency response-induced cross channel interference (CCI) between spectrally-overlaid orthogonal channels, a real-time, reconfigurable CCI cancellation (CCIC) function implemented in the OLT receiver DSP, is demonstrated to significantly improve the DFMA PON performance and robustness in terms of; significant reductions in total channel BERs and individual subcarrier BERs, substantial increases in channel capacities, considerably reduced sensitivity to ONU synchronisation and excellent transparency to the interfering channel's signal modulation format. The trade-off between the complexity of the CCIC function's filter, in terms of tap count (multiplier elements) and the DFMA PONs performance is optimised to determine the optimum trade-off. It is shown that the CCIC filter only requires as few as 42 taps to achieve excellent CCIC performance. We also fully analyse the DSP complexity of the transmit and receive DFMA filters and the CCIC filters for varying DFMA channel counts and varying ratios of $\mathrm{AD} / \mathrm{DA}$ converter sample rate to digital logic clock rate (parallelisation factor). It is shown that DFMA filter complexity is independent of channel count and furthermore that for channel counts $\geq 4$ the CCIC filter only needs to process $\sim 10$ interference-inducing samples per wanted signal sample, resulting in only $\sim 10$ multipliers per parallel filter in the CCIC filter. We have therefore successfully demonstrated an ultra-low complexity, real-time CCIC function, which considerably enhances the performance and robustness of DFMA PONs.
\end{abstract}

Index Terms - Optical fiber communication, Digital filter multiple access, digital signal processing (DSP), OFDM, 5G, passive optical networks (PONs).

\section{INTRODUCTION}

$\mathrm{T}$ o meet future data traffic demands it is now widely accepted that optical access networks must undergo a

Manuscript received February 12, 2019; revised May 17, 2019; accepted May XX, 2019. Date of publication May XX, 2019; date of current version May XX, 2019. This work was supported by the DESTINI project funded by the ERDF through the Welsh Government under the SMARTExpertise scheme. The work of E. Al-Rawachy was supported by the IRAQI Ministry of Higher Education and Scientific Research (MOHESR). (Corresponding author: Roger Philip Giddings.) fundamental transformation, as they must evolve into highly dynamic networks [1], providing high bandwidths, low latency [2], elastic network slicing [3], massive end-user connectivity and convergence of $5 \mathrm{G}$ mobile and fixed data traffic. The adoption of software defined networking (SDN), extended to the physical layer, for effective network control, is also critical for attaining highly efficient utilisation of the available dynamic network resources.

As passive optical networks (PONs) provide cost-effective access network solutions, to address the aforementioned technical requirements we have proposed and demonstrated a new PON architecture, termed digital filter multiple access (DFMA) PON [4,5], to provide a cost effective, SDNcontrolled and highly dynamic access network solution. The DFMA PON thus targets greatly increased operational flexibility in the access network physical layer, therefore expanding network reconfigurability and elasticity all the way to the network edge and so capitalising on the growing flexibility in metro and core networks. To dynamically share a common fiber transmission medium, the DFMA PONs employ SDN-controllable, DSP-based, digital orthogonal filters to dynamically manipulate multiple channels with elastic bandwidths and arbitrary signal modulations. Channel manipulation in the digital domain eliminates the need for expensive RF/optical components or multiple opticalelectrical-optical (O-E-O) conversions. Also importantly, the independent channels allowing key features such as i) converged fixed and mobile transport in a single PON, ii) ondemand, customer specific, virtual private networks (VPNs), and iii) network slicing which is a highly important feature needed to support the highly heterogeneous requirements of $5 \mathrm{G}$ services [6]. Other salient advantages of DFMA PONs can be summarized as follows:

- Extension of SDN control functionalities to the physical layer. Thus, drastically improving dynamic network reconfigurability, ease of network virtualisation and channel bandwidth elasticity.

- Inherent backwards compatibility with all existing PONs.

The authors are with the School of Computer Science and Electronic Engineering, Bangor University, Bangor, LL57 1UT, U.K. (e-mail: elp4b9@bangor.ac.uk; r.p.giddings@bangor.ac.uk; j.tang@bangor.ac.uk).E. Al-Rawachy is also with the College of Electronics Engineering, Electronics department, Ninevah University, Mosul, Iraq. 
- Transparency to underlying signal parameters such as modulation format.

- Enhanced data security in the physical layer due to the arbitrary nature of the digital filter configurations, i.e. full knowledge of the filter parameters is required to correctly demultiplex a channel from the aggregate signal.

- Inherent network scalability for "pay-as-you-grow" operation due to the ability to incrementally add capacity by exploiting a modular DSP solution in the optical line terminal (OLT).

- The low cost associated with large scale, mass-produced digital integrated circuits can keep PON capital expenditure (CAPEX) levels commercially viable.

A DFMA PON, however, can suffer severe performance degradation, mainly due to a non-flat physical channel frequency response with significant roll-off, which results in cross channel interference (CCI) [7,8]. Channel roll-off is determined by factors such optical fiber length, optical and RF component characteristics and the sinc response associated with zero-order hold DAC/ADCs. Hence, to maximize performance, a CCI cancellation (CCIC) technique that mitigates the channel frequency response roll-off effect can be highly beneficial.

Over the past few decades, various DSP-based interference cancellation (IC) techniques, predominantly targeted at cellular systems, have emerged. Parallel interference cancellation (PIC) $[9,10]$ and successive interference cancellation (SIC) [10] are the main categories of multi-user interference cancellation techniques. In the PIC process, the data symbols of all active users are initially detected in parallel, then the estimated interference signals are simultaneously subtracted from the received signal, data recovery is then performed again with reduced interference. The process can be repeated over several stages for enhanced IC performance. The SIC technique operates by detecting a single user's signal in consecutive stages, starting with the strongest signal, then the next strongest, and so on. When a signal is decoded, the interference it generates is estimated, based on the used modulation and channel estimate, this interference signal is then subtracted from the composite received signal for application to the next stage. PIC tends to be highly effective when all users are received with similar power levels, whereas SIC is superior when users have unequal received power levels. It may be possible to adapt the PIC and SIC techniques to address the CCI problem, however there are some fundamental disadvantages including, i) very high DSP complexity due to the need for full decoding of user signals and regeneration of the modulated signals, ii) high latency, especially in multi-stage designs, iii) dependency on signal modulation formats, and high sensitivity to channel estimation error [9]. On the other hand, least mean square (LMS)-based equalization [11] could also potentially be adopted to mitigate non-ideal physical channel frequency response-induced CCI. This technique employs an adaptive filter to equalize the non-flat channel response and thus dynamically minimise the received signal's error, the filter tap coefficients are iteratively updated based on a calculated error signal, a predefined step size and initial conditions, until they converge to a stable state that minimizes the error signal. However, there are potential drawback associated with the application of LMS-type algorithms in DFMA PONs, including i) a large number of iterations can be required to achieve convergence, which can result in unacceptably high network latency, especially when supporting latency critical 5G applications ii) high convergence sensitivity to initial conditions, which means convergence cannot always be guaranteed, and iii) the optimum tap count and step size can be dependent on system parameters such as bit rate and modulation format [11], and iv) the LMS algorithm attempts to optimize performance over the entire physical channel, whereas the channel to be equalized only operates in a limited spectral region of the physical channel.

Recently, we proposed and demonstrated a very simple but highly effective, DSP-based, CCIC technique to mitigate nonideal physical channel frequency response induced-CCI, which was experimentally demonstrated in a 2-channel, digital orthogonal filter multiplexed, point-to-point (PTP), standard single mode fiber (SSMF) transmission link of up to $36 \mathrm{~km}$ based on intensity modulation and direct detection (IMDD), using an offline-DSP based receiver [8]. The CCIC technique, which utilises a CCIC filter for interference signal estimation, has significant advantages in comparison to the aforementioned interference cancellation techniques due to its unique features such as, no initial condition dependency, transparency to signal modulation format and fast convergence as only a signal iteration stage is adequate [8]. It should be noted that the CCIC technique is, however, not restricted to IMDD-based systems and is compatible with coherent optical systems. The work was extended to a theoretical investigation of multi-channel interference cancellation in an IMDD DFMA PON using a modified technique termed the DFMA channel interference cancellation (DCIC) technique [12] that considers interference from all channels. The DCIC investigation showed that the cancellation of the interference between spectrum sharing optical network units (ONUs), i.e. orthogonal channels, is sufficient to achieve significant improvement in performance and that the total interference contributed from all remaining ONUs, operating in different spectral bands, is negligible in comparison [12]. Thus, CCIC is only essential between the orthogonal channels in a multi-channel IMDD DFMA PON system.

As it is vital to verify the practical implementation of DFMA PONs, in this paper, we report for the first time the experimental demonstration of a completely real-time DSPbased upstream DFMA PON based on IMDD. By employing two reconfigurable ONUs, we successfully demonstrate multiple upstream transmissions with various channel configurations, verifying that the real-time DSP-based orthogonal filters can be implemented with sufficient accuracy and operating speed, with software-controlled reconfiguration to dynamically select the ONU's operating channel(s). In the real-time IMDD DFMA PON, incorporating four independent OFDM-modulated channels, subject to CCI, we demonstrate the integration of our aforementioned CCIC technique, implemented in real-time DSP in the OLT, to verify its practical implementation and explore its performance under different DFMA PON channel configurations. The CCIC function is also fully optimised for low DSP complexity as it requires only 44 scalar multiplier elements, corresponding to only 11 multipliers per signal sample. The CCIC function is demonstrated to significantly improve the performance and robustness of the IMDD DFMA PON in terms of, i) significant improvements in 
BER versus received optical power, with the BER of individual OFDM subcarriers being reduced by factors as large as $100-$ 300 times, ii) significant increase in total channel capacity, of the order of $\geq 12$ times, iii) reduction of sensitivity to ONU synchronisation as the inter-ONU sample timing offset (STO) range can be increased by a factor of up to 15 times, and iv) excellent transparency to the interfering channel's modulation format, as negligible change in subcarrier BERs was observed when applying two different modulation formats on the interfering signal. Furthermore, we analyse the minimum DSP complexity requirements of the digital orthogonal filters and CCIC filters and show that their complexity does not scale with the implemented channel count. In addition, a crucial result for the CCIC filters is that each generated sample in the estimated interference signal only requires the processing of $\sim 10$ samples from the interference-inducing signal, such that only as few as $\sim 10$ scalar multipliers are required per parallel CCIC FIR filter. The results presented in this paper therefore verify that an ultralow complexity CCIC function is technically feasible and it leads to substantial improvements in the performance and robustness of IMDD DFMA PONs.

\section{DFMA PON OPERATING PRINCIPLE AND THE CCIC TECHNIQUE}

\section{A. DFMA PONS}

The IMDD DFMA PON operating principle is fully described in detail in [4], so here an outline of the basic principle is presented. The fundamental channel multiplexing is based on reconfigurable digital orthogonal shaping filters (SF), which divide the available signal bandwidth into multiple independent sub-wavelength bands, with two orthogonal channels, denoted as in-phase (I) and quadrature-phase (Q), occupying the same sub-wavelength band. All channels are thus independent and can carry arbitrarily modulated signals. To demultiplex the channels in the receiver a digital orthogonal matching filter (MF) is employed which is dynamically configured according to the targeted channel.

For the downstream direction, the orthogonal sub-band signals are digitally summed in the OLT to generate a multichannel aggregate signal, for digital-to-analogue conversion (DAC) before IMDD-based transmission. It is important to emphasise that, for correct downstream operation, appropriate ONU timing synchronisation is required in terms of absolute sample rate and STO.

For the upstream direction, assuming each ONU generates an optical signal consisting (typically) of a single orthogonal sub-band, the different channels are now optically combined in the PON's passive coupler. To ensure correct upstream operation, the timing synchronisation between orthogonal subbands is required to maintain orthogonality between the spectrally overlapped channels [5]. The OLT must therefore monitor and track timing synchronisation between each pair of orthogonal channels and use suitable control messages to periodically adjust the timing of an ONU's DAC in terms of sample rate and STO. The synchronisation between ONUs
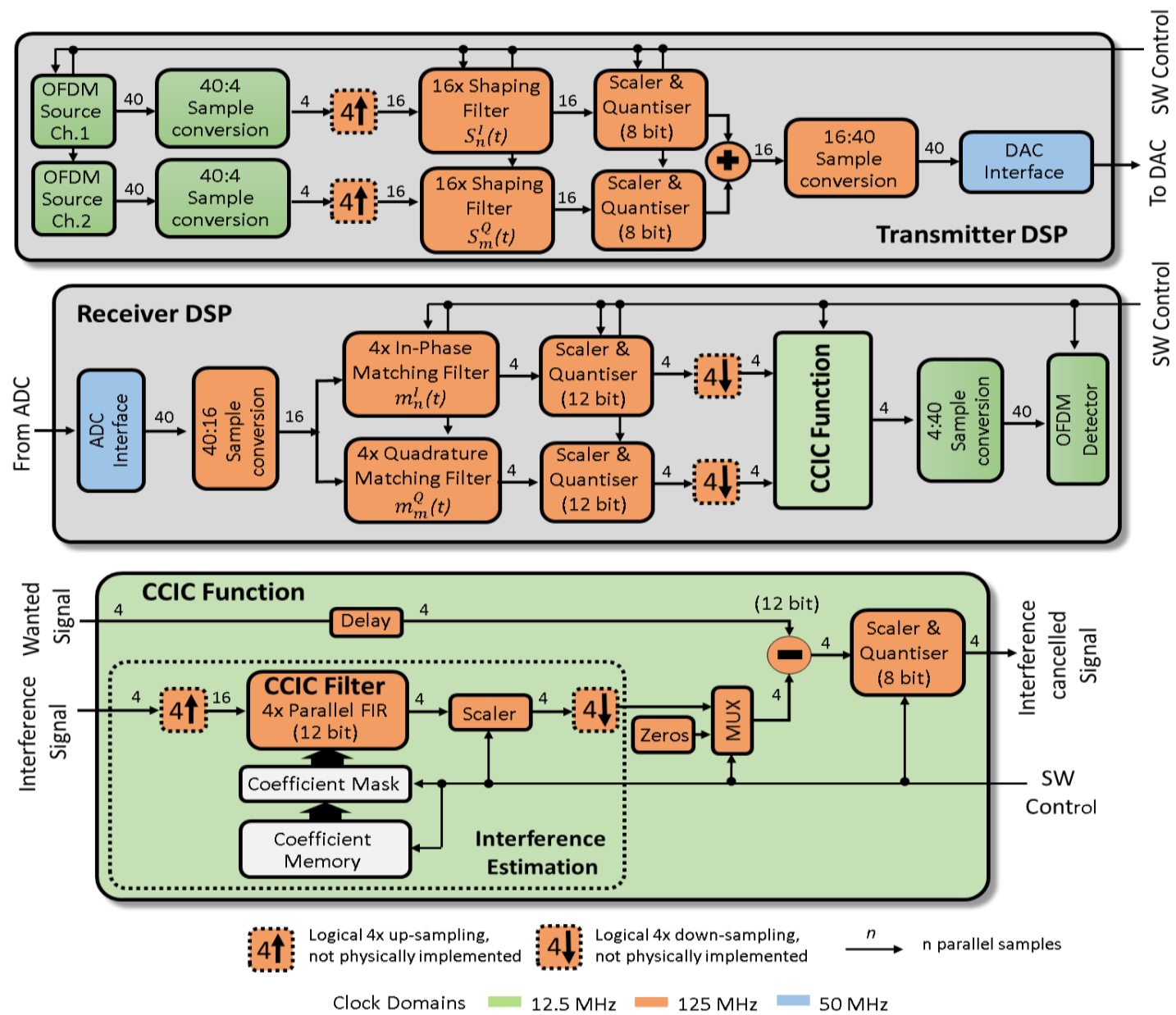

Fig. 1. Top level DSP architecture of the 4-channel CCIC-enabled DFMA PON transceiver 
operating in different sub-wavelength bands is not needed for the purpose of maintaining channel orthogonality, however if the OLT employs a common ADC to receive all channels then the timing of all channels and therefore ONUs, must be aligned [7]. It is possible for an ONU to implement multiple SFs/MFs for multi-channel operation, and multiple ONUs can also share the same physical channel in the time domain [13]. Furthermore, an ONU can achieve a variable asymmetric transmission capacity as the upstream and downstream directions are independent as so can use independent filter configurations.

As the overall configuration of the DFMA PON is dependent on reconfigurable DSP functions, a centralized SDN-controller can communicate, via a protocol such as extended OpenFlow, with a transceiver-embedded DSP controller to dynamically configure the DFMA PON in terms of the characteristics of the involved digital filters and/or channels, to allocate the channels at their optimum spectral locations and with required bandwidths. Thus highly reconfigurable, dynamic and elastic bandwidth provision is achievable in the DFMA PON [4].

\section{B. Cross-channel interference and the cancellation technique}

As previously stated, the DFMA PON suffers from CCI [8] between orthogonal sub-bands due to a non-ideal physical channel response, the CCI resulting in a degradation in PON performance. The transmitter's up-sampling function combined with the SF, generates a double sideband (DSB), sub-band signal, which suffers an imbalance in sideband power when subject to a transmission channel with a roll-off in its frequency response. When a MF removes an unwanted orthogonal channel, it relies on sideband cancellation, thus the sideband power imbalance leads to CCI. Consequently, the more severe the channel roll-off effect the greater the CCI levels [8].

Full details of our proposed CCIC technique and the detailed fundamental theory are presented in [8], where a two channel, PTP optical link, employing off-line signal processing was demonstrated. The basic CCIC principle employs an interference signal path emulating CCIC filter. The received, interference-generating signal is applied to the CCIC filter to obtain an interference signal estimate, which is subsequently subtracted from the wanted recovered signal to achieve interference cancellation. As the CCIC filter emulates the response of a full interference path, it corresponds to the concatenation of the unwanted channel's SF, the estimated physical channel and the wanted channel's MF. As the SF and MF responses are known, the physical channel response must be periodically estimated in a practical transceiver, in order to dynamically determine the required CCIC filter responses. The CCIC function also incorporates the up/down-sampling functions before/after the CCIC filter, as they are included in the interference signal path. Appropriate, fixed, signal scaling and delays are also applied to align the actual and estimated interference. Furthermore, the accuracy of the implemented CCIC filter will obviously impact the accuracy of the interference estimate and therefore the levels of any residual CCI.

\section{REAL-TIME DFMA PONS INCORPORATING LOW COMPLEXITY CCIC}

\section{A. DSP architecture of CCIC-enabled real-time transceivers}

Fig. 1 shows the top-level DSP architecture for the optical transceiver's transmitter and receiver elements, which are implemented in field programmable gate arrays (FPGAs). The presented architecture shows the implemented DSP, which is based on the reconfigurable transceiver described in [14], which supports a two-channel system with Hilbert pair-based digital orthogonal filters for channel multiplexing. The transceivers employed here are designed to support a 4-channel system so an up-sampling factor of 4 is employed. An adaptive OFDM signal source [15], with PRBS test data and key parameters as specified in Table. I, provides a data signal for each channel. As

Table I. Transceiver and system parameters

\begin{tabular}{cc}
\hline Parameter & Value \\
\hline OFDM IFFT/FFT size & 32 points \\
Maximum data carrying OFDM subcarriers per channel & 15 \\
Subcarrier frequency spacing & $15.625 \mathrm{MHz}$ \\
Subcarrier modulation format & $16-\mathrm{QAM}$ \\
DAC and ADC sample rate / bit resolution & $2 \mathrm{GHz} / 8 \mathrm{bits}$ \\
OFDM encoder/decoder clock rate & $12.5 \mathrm{MHz}$ \\
Data sequence length for error counting & $88,500 \mathrm{Symbols}$ \\
Samples per symbol* IFFT/Cyclic prefix/Total & $32 / 8$ / 40 Samples \\
Line rate per channel (13 subcarriers enabled) & $812.5 \mathrm{Mb} / \mathrm{s}$ \\
Adopted FEC limit & $1 \mathrm{E}-3$ \\
EML wavelength/modulation bandwidth & $\sim 1550 \mathrm{~nm} \& / 10 \mathrm{GHz}$ \\
EML laser bias current/EAM bias voltage/MZM DC bias & $125 \mathrm{~mA} /-0.75 \mathrm{~V} / 1.43 \mathrm{~V}$ \\
EML/MZM driving voltage & $2 / 1.2 \mathrm{Vpp}$ \\
TLS wavelength/MZM modulation bandwidth & $\sim 1550 \mathrm{~nm}{ }^{\&} / 20 \mathrm{GHz}$ \\
EML/MZM optical launch power before EDFA & $2.6 /-2.3 \mathrm{dBm}$ \\
EML and MZM optical launch power after EDFA & $4.5 \mathrm{dBm}$ \\
Electrical signal amplitude at ADC & $340 \mathrm{mVpp}$ \\
PIN bandwidth/noise & $12.5 \mathrm{GHz} / 2.8 \mathrm{mVrms}$ \\
PIN responsivity at 1550nm & $0.55 \mathrm{~A} / \mathrm{W}$ \\
SSMF length & $26 \mathrm{~km}$ \\
\hline
\end{tabular}

* Before up-sampling and after down-sampling

$\&$ Wavelength spacing $=0.3 \mathrm{~nm}$ 
the employed DAC operates at $2 \mathrm{GS} / \mathrm{s}$, the SF consists of 16 parallel FIR filters, with software-controlled tap coefficients, operating at $125 \mathrm{MHz}$ to achieve the required sample throughput. An on-line adjustable scaling function with 8-bit quantisation follows the SF to allow signal power adjustment. Two channel instances are implemented in a single transmitter, with their outputs digitally summed, before application to the DAC interface. As the 8-bit, filter tap coefficients are softwarecontrolled each SF can be dynamically configured to support any one of the four possible channels. Full details of the SF DSP design for low complexity are discussed in section V-B.

As the DAC/ADC sample rate is $2 \mathrm{GS} / \mathrm{s}$, the four transmitter generated channels are an $\mathrm{I}(\mathrm{Q})$ channel in the baseband region from $0-0.5 \mathrm{GHz}$ designated as $\mathrm{BB}-\mathrm{I}$ (BB-Q) and an $\mathrm{I}(\mathrm{Q})$ channel in the passband region from $0.5-1 \mathrm{GHz}$ designated as PB-I (PB-Q). The inset in Fig. 2 shows example electrical spectra at the ONUs of the two sub-wavelength bands.

The architecture of the receiver's DSP is also based on that presented in [14], however here the design supports 4 channels and two FIR-based, reconfigurable, MFs are implemented. Full details of the low complexity, MF DSP design are discussed in in section V-B. The first MF recovers the wanted signal and the second MF recovers the interference-generating signal in the corresponding orthogonal channel. For demonstration purposes only one OFDM decoder function is implemented to decode and analyse the BER of the wanted, CCIC-compensated, channel, however in a practical implementation both orthogonal channels could be recovered. The recovered interferencegenerating signal is applied to the CCIC function, as illustrated in Fig. 1, where, after suitable up-sampling, it is applied to the CCIC filter, suitably scaled and down-sampled to generate an estimate of the interference signal. The 12-bit, estimated interference signal is then subtracted from the 12-bit, suitably delayed wanted signal, before signal decoding, channel/subcarrier BER analysis and subcarrier constellation observation in the OFDM decoder function. The CCIC function operates with 12-bit samples as this resolution limits complexity and minimises the quantisation noise effect to achieve good CCIC performance at the lower interference levels present at the higher signal frequencies
The CCIC function also incorporates additional functionality for the purpose of performance analysis only. Firstly, the interference cancellation can be disabled via a multiplexer, shown in Fig. 1, which allows the interference estimation signal to be set to zero-valued samples thus allowing the system performance to be observed without CCIC enabled. Secondly, as it is important to optimise the complexity of the CCIC filter to minimise implementation cost and power consumption, whilst maintaining sufficient performance, the CCIC filter design incorporates a coefficient mask located between the 64-tap coefficient memory and the CCIC filter coefficient inputs. This software-controlled mask can dynamically limit the effective filter tap count by setting the unwanted tap coefficients to zero. This allows full investigation of the trade-off between complexity (tap-count) and performance. The coefficient mask is designed to limit CCIC filter tap count to $64,54,42,32,16$ or 8 .

\section{B. Experimental System Setup}

The upstream DFMA PON experimental system setup and the associated transceiver/system parameters are given in Fig. 2 and Table. I, respectively. The more challenging upstream transmission is experimentally demonstrated as it involves the optical summation of different channels with timing synchronisation necessary between ONUs to maintain channel orthogonality. The setup consists of two ONUs (with dualchannel transmitters) and one OLT (with a single-channel receiver). Both ONUs, incorporating the aforementioned DSP and DACs, include an RF delay line for fine adjustment of signal timing, an RF gain stage based on a fixed gain RF amplifier and variable gain electrical attenuator, an intensity modulator (IM) for E/O conversion, and an EDFA for optical power boost and control with a tuneable $0.8 \mathrm{~nm}$ OBPF to minimise out-of-band, amplified spontaneous emission noise (ASE). The IM in ONU1 is an electro-absorption modulated laser (EML) which integrates a DFB laser, as its light source, with an electro-absorption modulator (EAM), both of which are subject to optimised operating conditions including DC biasing, temperature control and driving voltage as specified in Table. I. The IM in ONU2 consists of a tuneable laser source (TLS) and

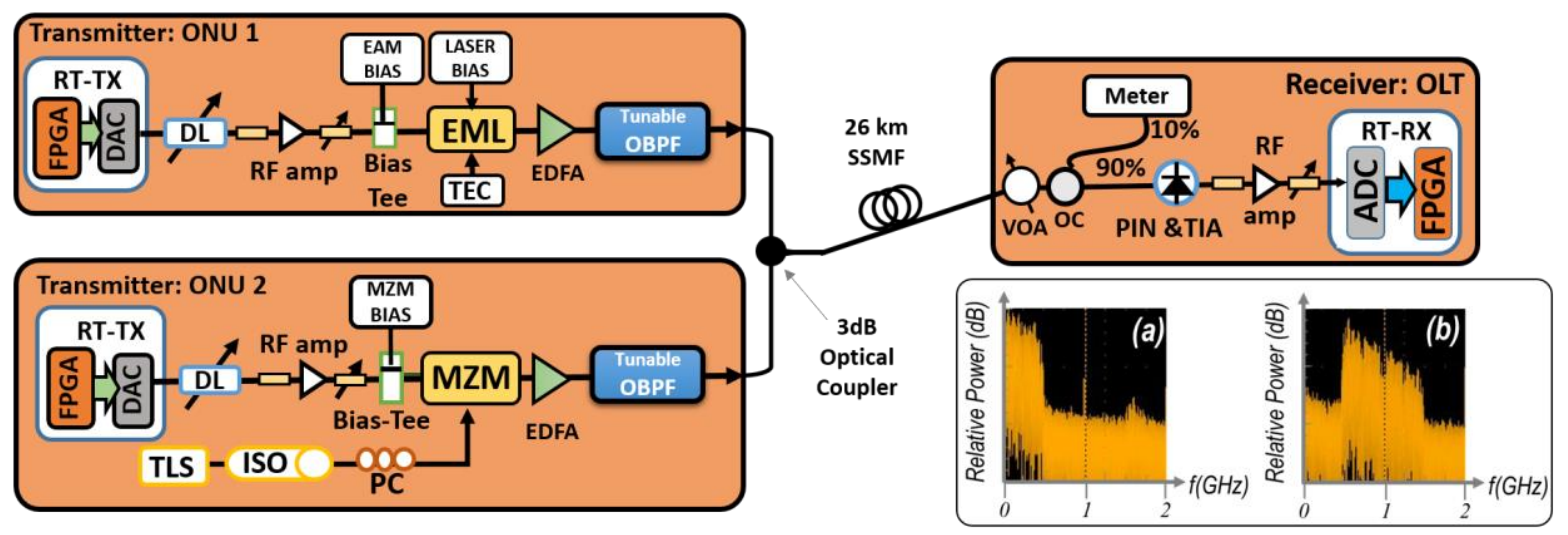

Fig. 2. Real-time DFMA PON upstream experimental system setup. Inset: Example electrical spectra at the ONUs with all subcarriers enabled (a) BB-I+BB-Q, (b) PB-I+PB-Q. Note: Image spectra shown from 1-2GHz.

DL: Delay line; EML: electro-absorption modulated laser; TEC: thermo-electric controller; MZM: Mach-Zehnder modulator; VOA: variable optical attenuator; OBPF: optical bandpass filter; TLS: tunable laser source; PC: polarization controller; PIN+TIA: photodetector with integrated transimpedance amplifier; EDFA: erbium doped fiber amplifier; ISO: Isolator; RT-TX: real-time transmitter; RT-RX: real-time receiver 
a Mach-Zehnder modulator (MZM). The TLS output passes via an isolator and an optimally adjusted polarization controller prior to feeding the MZM input. The MZM is biased at its quadrature point. During MZM operation, the bias voltage and driving voltage amplitude are fine-tuned for best performance. The output powers of both EDFAs are adjusted to $4.5 \mathrm{dBm}$. The optical signals from the ONUs are combined in a $3 \mathrm{~dB}$ passive optical coupler, representing the remote node of the PON, before the optically combined signal is launched into a $26 \mathrm{~km}$ SSMF. Both ONUs operate at a nominal wavelength of $1550 \mathrm{~nm}$, however a wavelength spacing of $0.3 \mathrm{~nm}$ is employed to avoid the direct-detection (DD)-induced optical beat interference (OBI) effect [16], in practice wavelength control can be achieved by laser thermal detuning [17] or alternative OBI mitigation methods [18-19] may be employed after suitable modifications. For experiments involving a PTP configuration, ONU1 and the $3 \mathrm{~dB}$ optical coupler are omitted so that ONU2 connects directly to the $26 \mathrm{~km}$ SSMF link.

The OLT-based receiver consists of a variable optical attenuator (VOA) to adjust the received optical power (ROP) level, a 90:10 optical splitter for ROP measurement, a $12.5 \mathrm{GHz}$ linear PIN photodetector with integrated transimpedance amplifier (TIA) for direct detection of the optical signal, an RF gain stage, similar to that employed in the ONUs, to optimise the analogue signal level, a $2 \mathrm{GS} / \mathrm{s}, 8$-bit ADC and finally an FPGA for the receiver DSP operations. It should be noted that the ADC incorporates a suitable antialiasing filter to remove out-of-band receiver noise before signal sampling.

The frequency responses of the two paths, i.e. from ONU1 to the OLT employing the EML and from ONU2 to the OLT employing the MZM, are measured using a pilot tone-based channel estimation method. This provides the discrete channel frequency response coefficients from $62.5 \mathrm{MHz}$ to $937.5 \mathrm{MHz}$ in $62.5 \mathrm{MHz}$ intervals. The discrete channel impulse responses are then computed for use in the CCIC filters. Both paths show
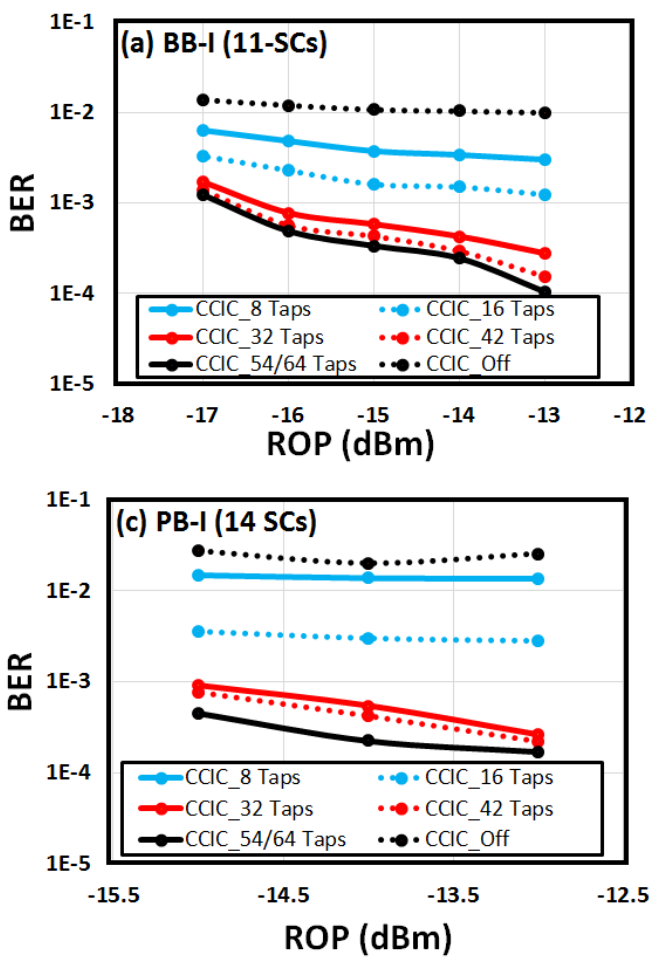

a reasonably similar frequency response with a total roll-off of $10.5-12 \mathrm{~dB}$ over the $1 \mathrm{GHz}$ spectral region, therefore, the same channel impulse response is utilized when determining the four different CCIC filter responses corresponding to the four channels. In addition, as the level of frequency response roll-off is relatively high, it has a high probability of representing worstcase practical scenarios, thus providing an excellent environment for evaluating the CCIC technique's performance.

\section{Performance of low complexity CCIC in point-to-point SMF links}

To verify the performance of the real-time CCIC function and investigate the impact on performance of CCIC filter tap count, a $26 \mathrm{~km}$ SSMF PTP optical link is adopted as described in section III-B, and shown in Fig. 2, with the parameters specified in Table. I. Thus the PTP link uses ONU2 with a TLS and MZM for optical intensity modulation. The PTP link is adopted as it allows reliable evaluation of the CCIC function's performance by eliminating any possible inter-ONU STO-induced channel leakage [12] caused by non-optimised inter-ONU synchronisation. To assess the performance of the real-time CCIC function, BER versus ROP measurements are performed. The adopted measurement procedure is as follows; the OFDM subcarriers are set for 16-QAM modulation with a flat subcarrier power loading profile, then with only one sub-band channel enabled all parameters are optimised (as in Table. I) for minimum channel BER. To optimise the receiver's sample timing, whole sample interval adjustments are made in the ONU's OFDM encoder and sub-sample interval adjustments are made with the manual RF delay line in the ONU. All transceiver and system parameters are thus optimised for a minimum channel BER. For the BB (PB) sub-wavelength band only the highest frequency 11 (14) subcarriers are enabled as the 3 (1) lower frequency subcarriers have BERs in excess of the forward error correction (FEC) limit. When the first sub-
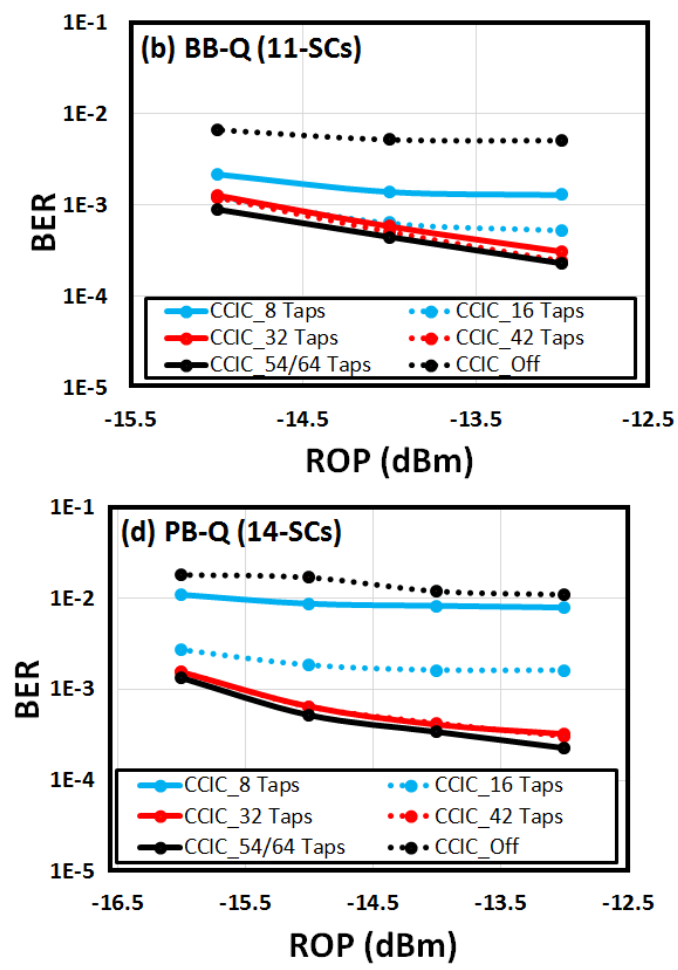

Fig. 3. BER vs. ROP for (a) BB-I, (b) BB-Q, (c) PB-I, (d) PB-Q for varying CCIC filter complexity for pointto-point $26 \mathrm{~km}$ SSMF transmission 
band channel is operational, the corresponding orthogonal subband channel is then enabled and parameters such as RF gain and digital scaling values are adjusted to minimise the total BERs of both channels simultaneously. Fig. 3 shows measured BER versus ROP curves for each of the four channels with a different number of CCIC taps enabled and with the CCIC function disabled.

The BER measurements in Fig. 3 clearly show that when no CCIC is applied, there are significant levels of CCI, which prevents all channels from achieving a BER below the FEC limit, regardless of the ROP level, as indicated by the flat characteristic of the BER curves. However, the real-time CCIC function substantially improves BER performance to acceptable levels on all channels when enabled with a sufficient number of filter taps. Fig. 3 clearly shows that in all cases, a minimum of 32 taps are required to achieve a BER below a FEC limit of $1 \times 10^{-3}$ (corresponding to $7 \%$ overhead [20]). To allow some margin however, 42 taps can be considered to provide a good trade-off between the CCIC filter complexity and the channel performances. It is also interesting to note that the general BER developing trends observed in Fig. 3, of very shallow curves with CCIC off and steeper curves with CCIC on, is also observed in both the offline [8] and theoretical [12] CCIC results.

\section{Performance of low complexity CCIC in DFMA PONs}

Having validated the CCIC function in a PTP optical link, it is essential to verify its operation in a practical IMDD DFMA PON scenario. For this experiment, the setup in Fig. 2 is adopted with all associated parameters set as specified in Table. I, and the CCIC filter tap count is set to the optimum value of 42 taps as determined in section III-C. The ONUs are configured such that ONU2 (ONU1) generates channels BB-Q and PB-I (BB-I and PB-Q), this provides a worst case channel configuration scenario as inter-ONU STO timing must be correctly adjusted to maintain orthogonality between channels in the same sub-wavelength band. All OFDM subcarriers are
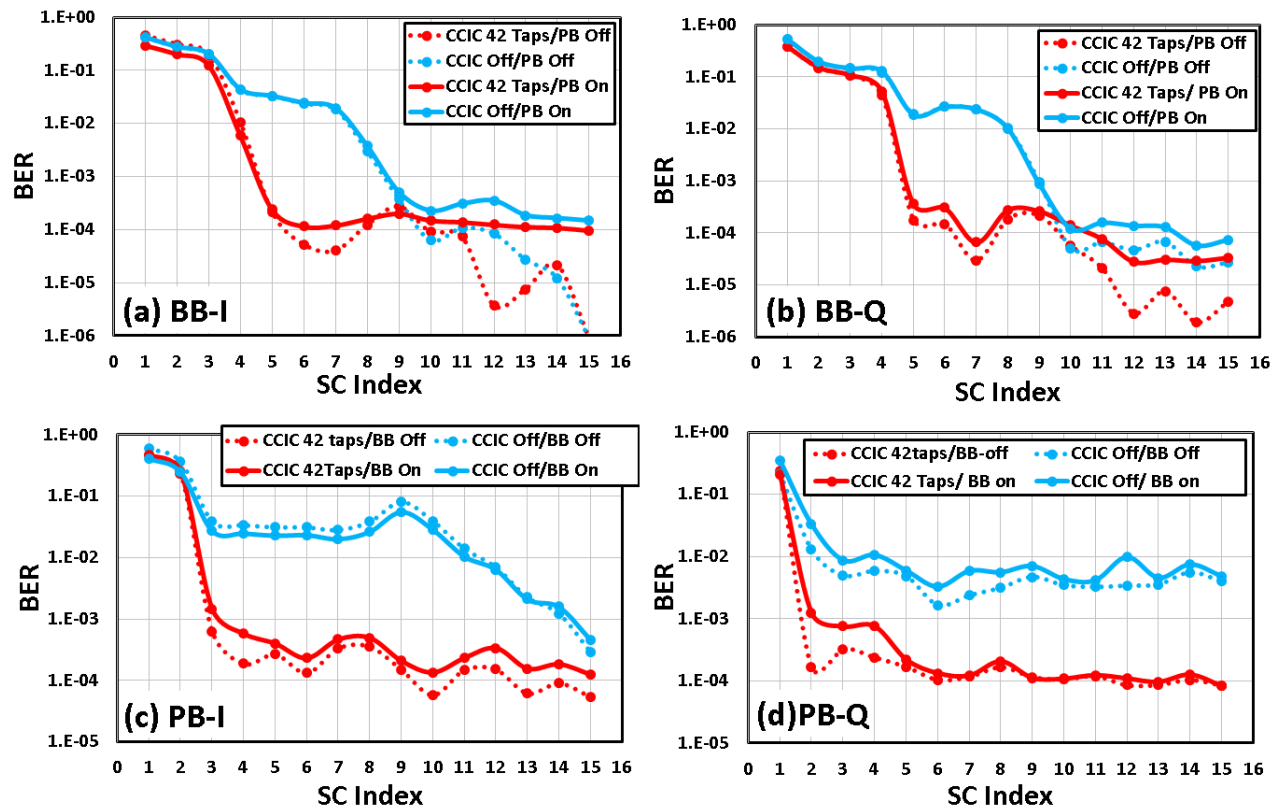

Fig. 4. BER vs. subcarrier index for $26 \mathrm{~km}$ SSMF DFMA PON transmission at a total ROP of $-10 \mathrm{dBm}$ with 42 tap CCIC filters a) BB-I, b) BB-Q, c) PB-I and d) PB-Q enabled in order to observe the CCIC function's effectiveness across the entire signal spectral range. The total ROP is set to $10 \mathrm{dBm}$, which corresponds to a ROP for each ONU of $-13 \mathrm{dBm}$.

The method adopted to configure the system for optimum upstream performance is as follows: firstly, only ONU1 is enabled and configured to generate channels BB-I and PB-Q, this logical PTP set up is optimised, as described in section III$\mathrm{C}$, following the same procedure for receiver sample timing adjustment. Secondly, ONU2 is enabled and configured to generate the BB-Q and PB-I channels. The integer valued, sample timing offset is adjusted in the OFDM encoder in ONU2 to ensure the correct samples are recovered in the receiver and the inter-ONU STO is adjusted via the manual RF delay line in ONU2. Finally, to simultaneously minimise the BERs of all channels the RF gains and SF/MF digital scaling values are adjusted as necessary.

To receive a specific channel the MFs and CCIC filter in the receiver are configured with the appropriate impulse responses. The real-time BER of each individual subcarrier within a channel is then observed in order to plot the BER versus subcarrier index for each channel, as shown in Fig. 4. To observe the effectiveness of the CCIC function on each channel, the CCIC is disabled, whilst keeping all other parameters unchanged, and the BER versus subcarrier index for this case is measured as shown in Fig. 4. Furthermore, to observe the impact on BER performance of the neighbouring subwavelength band, for each channel the BER versus subcarrier index is plotted with the 2 channels in the neighbouring subwavelength band disabled, for the cases of CCIC enabled and disabled, as shown in Fig.4.

The observed results verify that the CCIC function, implemented with only a 42 tap filter, is highly effective in removing CCI between orthogonal channels in the $26 \mathrm{~km}$ SSMF DFMA PON. When considering the channel capacity in terms of the number of subcarriers with BERs below $1 \times 10^{-3}$, the observed CCIC-induced increases in channel capacity for the 
cases of CCIC off and CCIC on are illustrated in Table. II. It

Table II. Working subcarrier count with and without CCIC enabled

\begin{tabular}{cccc}
\hline Channel & $\begin{array}{c}\text { SCs when } \\
\text { CCIC off }\end{array}$ & $\begin{array}{c}\text { SCs when } \\
\text { CCIC on }\end{array}$ & $\begin{array}{c}\text { Capacity } \\
\text { Increase } \\
\text { Factor }\end{array}$ \\
\hline BB-I & 7 & 11 & 1.6 \\
BB-Q & 7 & 11 & 1.6 \\
PB-I & 1 & 12 & 12 \\
PB-Q & 0 & 13 & $\infty$ \\
\hline
\end{tabular}

would also be possible in practice for an ONU, to further maximise the capacity of its dynamically allocated and hence variable channel, by exploiting the adaptive bit and power loading feature of OFDM.

It can also be inferred from Fig. 4 that the dominant CCI occurs between spectrally overlapped orthogonal channels as there is minimal reduction in subcarrier BERs when the neighbouring sub-wavelength band is turned off, hence, there is a finite, yet very low level of interference from a channel's adjacent sub-wavelength band, which is due to the large, but finite, out-of-band attenuation of the digital filters. This important result is in full agreement with the results from the numerically investigated DCIC technique presented in [12].

When considering the BER vs. subcarrier index curves in Fig. 4(a-d), for the case of CCIC disabled, there is a general trend of lower frequency subcarriers suffering higher BERs, which is consistent with the fundamental CCI mechanism outlined in section II-B, as the lower frequency subcarriers are located at the outer edges of the sidebands and so suffer more from the sideband power imbalance effect. Also, the frequency response profile for the $\mathrm{PB}$ channels has a higher roll-off ( $7 \mathrm{~dB})$ compared to the $\mathrm{BB}$ channels $(\sim 4 \mathrm{~dB})$ which explains why the $\mathrm{PB}$ channels, in comparison to the $\mathrm{BB}$ channels, have virtually no subcarriers with a BER below the FEC limit for the CCIC-disabled cases. The general variation between the curves can be due to the fact that the CCI levels are dependent on the specific channel frequency response profiles. When considering the CCIC enabled case, the curves show a trend of a reasonably flat BER but rising sharply at the lower frequency subcarriers, the flatness indicating that the CCIC is equally effective for different levels of CCI. The aforementioned sharp rise in BER can be attributed to the finite tap count-induced enhancement of digital filter frequency response ripples, which leads to higher attenuation at the filter edges, where the low frequency subcarriers are located. Furthermore, unwanted subcarriersubcarrier-intermixing (SSI) products [21] are generated upon square-law photon detection, where characteristically the noise power is higher at lower signal frequencies, thus the impact of SSI is more severe in the BB region, which can explain why the BB curves show high BERs at more of the lower frequency subcarriers. The shape of the curves with CCIC enabled is also dependent on the accuracy of the channel estimation used in the CCIC function. However, an important result shown in Fig. 4, is that the CCIC function significantly improves the BER of a substantial number of subcarriers by factors of up to $\sim 360$, interestingly this is of a similar order to that observed in the offline CCIC experiment in [8].

In the off-line CCIC experiment in [8], which uses a very similar optical link between the ONU and OLT, hence has a similar physical channel response, the BER versus subcarrier index curves show a general trend of CCIC performance increasing with subcarrier frequency, however a similar trend is not apparent in the plots in Fig. 4. This can be due to differences in the sub-wavelength band frequency response profiles [7], as here (in [8]) each sub-wavelength band occupies half (all) of the $1 \mathrm{GHz}$ aggregate signal bandwidth. Also, the real-time DSP hardware employs fixed-point calculations (max 12 bit) in comparison to the high precision, floating-point calculations of the off-line processing approach. This can reduce the CCIC effectiveness at low CCI power levels, nevertheless, the finite precision of the real-time DSP implementation is demonstrated to be sufficient to achieve highly effective CCIC.

\section{REAL-TIME DFMA PON PERFORMANCE}

To further explore the effectiveness of the CCIC function and its impact on system robustness, in this section further analyses is undertaken of the performance of a real-time DFMA PON incorporating low complexity CCIC, in terms of BER performance, impact on tolerance to inter-ONU STO and transparency of the CCIC function to the interfering signals modulation format.

\section{A. Low complexity CCIC-induced improvement in DFMA PON performance}

The IMDD DFMA PON system employing a 26km SSMF link, as described in section III-D, is employed with ONU1 (ONU2) generating the PB (BB) sub-wavelength bands. The BER performance of both the PB-I and PB-Q channels, with BB-I and BB-Q channels being present, is measured with 13 subcarriers enabled ( $3^{\text {rd }}$ to $15^{\text {th }}$ ) allow a fair comparison and ensure all subcarriers operate with BERs below the adopted FEC limit of $1 \times 10^{-3}$. Small adjustments to the subcarrier power loading profiles are made to reduce the BER of low frequency subcarriers with BERs close to the FEC limit. BER measurements are performed with and without the 42-tap count CCIC function enabled, and to further verify that 42 taps are adequate, comparative BER measurements are also performed with 54 and 64 taps enabled. Fig. 5(a) shows the BER versus ROP for the PB-I channel, in the region of the adopted FEC limit, for the aforementioned cases and Fig. 5(b) shows the corresponding measurements for the PB-Q channel. The BB-I and BB-Q channel performances are not shown in Fig.5 as their performances are similar to the PB-I and PB-Q channels. It is clear that without CCIC the total channel BERs greatly exceed the FEC limit, whereas when CCIC is enabled with 42 taps, both channels can operate below the FEC limit when the total ROP level is $\geq-13.4 \mathrm{dBm}(\geq-13.2 \mathrm{dBm})$ for the PB-I (PB-Q) channel. Considering ROP per ONU, to allow comparison with the PTP case, the corresponding ROPs for PB-I (PB-Q) at the FEC limit are $-16.4 \mathrm{dBm}(-16.2 \mathrm{dBm})$, which are $\sim 1 \mathrm{~dB}$ lower than the corresponding PTP case, this can be due to the fact that, in the DFMA PON case a second, high BER, subcarrier has been dropped. This fact can also be the reason for the increased slope of the BER curves. It is also evident that there is now only an extremely small variation in performance between the 64,54 and 42 tap count cases, which is smaller than that observed in the corresponding PTP case plotted in Fig. 3. This can also be due to the aforementioned dropping of the low performance $2^{\text {nd }}$ 

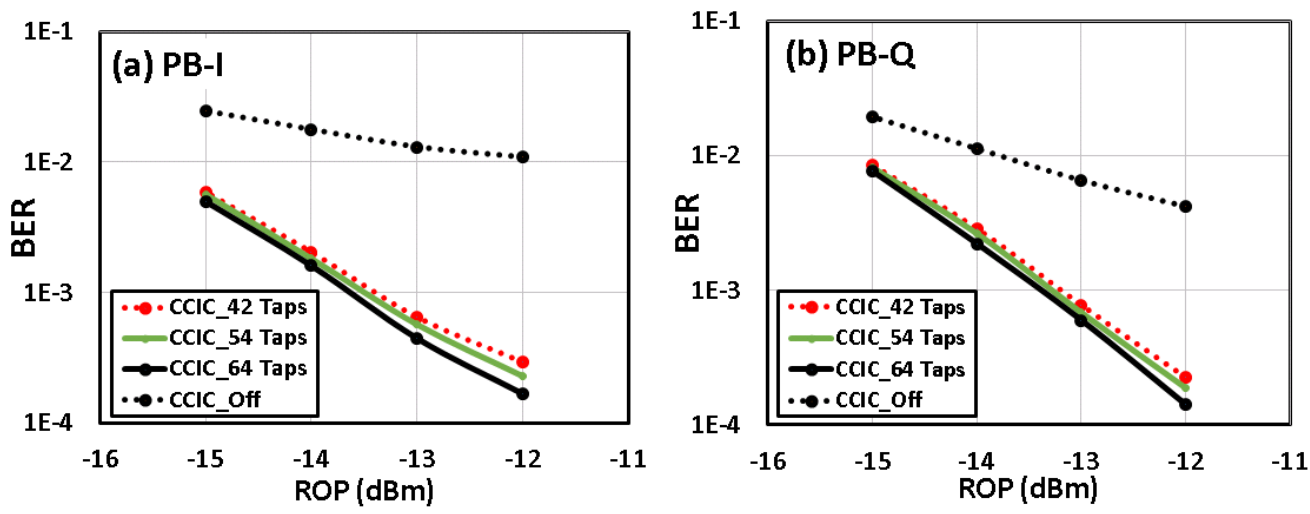

Fig. 5. BER vs. ROP for passband channels using varying CCIC filter complexity for 26km SSMF MPTP transmission using 13 subcarriers (a) PB-I (b) PB-Q
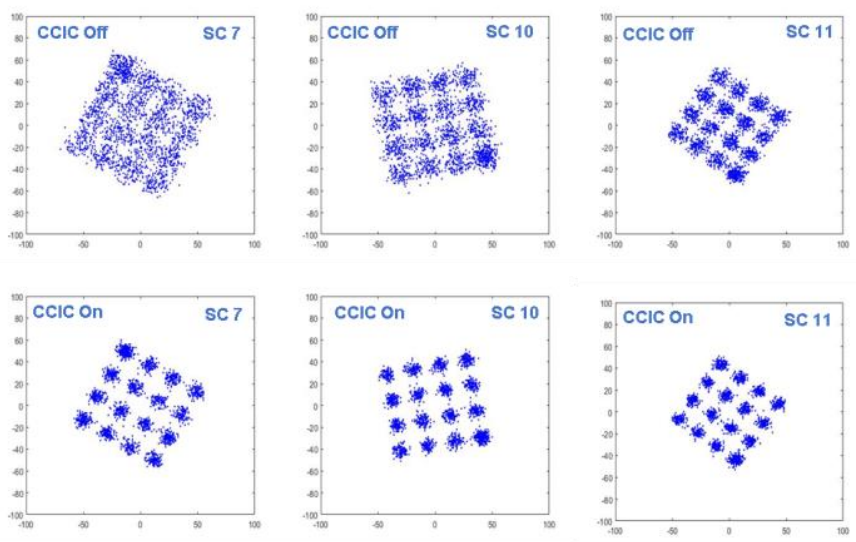

Fig. 6. Example unequalised PB-I subcarrier constellations for the cases of CCIC disabled and enabled in a 25km SSMF DFMA PON at -10dBm total ROP and 42 tap CCIC filters. (SC: subcarrier)

subcarrier. Fig. 5, thus further validates 42 taps as the optimum CCIC filter tap count. As 16-QAM is adopted on each subcarrier the total line rate of each channel is $812.5 \mathrm{Mb} / \mathrm{s}$, or $1.625 \mathrm{~Gb} / \mathrm{s}$ aggregate line rate for the PB subwavelength band, corresponding to a spectral efficiency of $3.25 \mathrm{~b} / \mathrm{s} / \mathrm{Hz}$. Hence, an aggregate signal bandwidth of $15 \mathrm{GHz}$ could support $\sim 50 \mathrm{~Gb} / \mathrm{s}$.

For the PB-I channel, Fig. 6 shows example unequalised subcarrier constellations for the cases of CCIC enabled and disabled, for the conditions corresponding to the measurement case in Fig. 5(a) and a total ROP of $-10 \mathrm{dBm}$. As would be expected, the constellations with CCIC enabled show a clear reduction in CCI compared to the case when CCIC is disabled, which is fully consistent with the observed reductions in the subcarrier BERs. It is also observed that each of the 16-QAM constellation points has a more square (round) shape for the case of CCIC disabled (enabled). This is due to the fact that, the $\mathrm{CCI}$ at a specific subcarrier frequency originates from the corresponding spectrally overlapped subcarrier in the orthogonal sub-band, which has a characteristic square shape due to the square-shaped 16-QAM constellation. Whereas when CCIC is enabled, the predominant noise is additive white Gaussian noise (AWG) from sources such as the PIN photodetector and the RF amplifiers and so it generates the characteristically rounded constellation points.

\section{B. Impact of Real-Time CCIC on Inter-ONU STO Tolerance}

Achieving suitable timing synchronization between ONUs occupying the same signal spectral region is essential for the DFMA PON operation. Therefore, the effect of the CCIC function on the tolerance of the DFMA PON to inter-ONU STO is explored in this section. The PON system employing a $26 \mathrm{~km}$ SSMF link, as described in section III-D, is employed with ONU1 (ONU2) generating the BB-I and PB-Q (BB-Q and PBI) channels, such that orthogonal sub-bands originate from different ONUs. To allow inter-ONU STO range comparisons for the cases of CCIC enabled and disabled, and as the range is dependent on the number of enabled subcarriers and the ROP, only the 6 highest frequency subcarriers are employed in each channel with ROP set to $-9 \mathrm{dBm}$, to ensure channel BERs below the FEC limit of $1 \times 10^{-3}$ can still be achieved when the CCIC function is disabled. All subcarriers adopt 16-QAM modulation and all other parameters are as specified in Table. I. The CCIC function also employs the previously identified optimum tap count of 42. Here, the inter-ONU STO range is defined as the maximum signal time delay variation range of the ONU with varied sample time, which maintains the BER of the ONU with fixed sample time, below the adopted FEC limit, thus, loss of channel orthogonality is purely generated by inter-ONU STO as receiver STO is maintained at the optimum value. To measure the inter-ONU STO range, the inter-ONU STO between the transmitters in the ONUs and the receiver STO at the OLT are optimised for minimum BER on all channels. Then, using the RF delay lines the signal timing of ONU1 (ONU2) is advanced and delayed in relation to its optimum value in steps of $12.5 \mathrm{ps}$, whilst the BER of PB-I (BB-I) is monitored.

Fig. 7(a) shows the BER variation of the BB-I channel from ONU1, as the timing of the BB-Q channel from ONU2 is varied for the cases of CCIC enabled and disabled. In the figure, Ons corresponds to the optimum ONU synchronisation case. A significant increase in inter-STO timing range is achieved from $0.04 \mathrm{~ns}$ with CCIC off to $0.2 \mathrm{~ns}$ with CCIC on, corresponding to a five-fold increase. Fig. 7(b) shows the corresponding case for the PB-I channel from ONU2 when the timing of the PB-Q channel from ONU1 is varied, here an approximate fifteen-fold increase in inter-STO range is achieved from $\sim 0.01 \mathrm{~ns}$ for CCIC off to $0.15 \mathrm{~ns}$ for CCIC on. The PB-I channel is observed to be more sensitive to the inter-ONU STO compared to the BB-I channels, which is expected as the PB channels operate at higher frequencies where a given time offset results in a greater signal amplitude deviation. The CCIC-induced improvements in inter-ONU STO ranges now significantly relaxes the ONU synchronisation requirements as the ranges now correspond to $40 \%(30 \%)$ of the 500ps sample interval for BB-I (PB-I). 

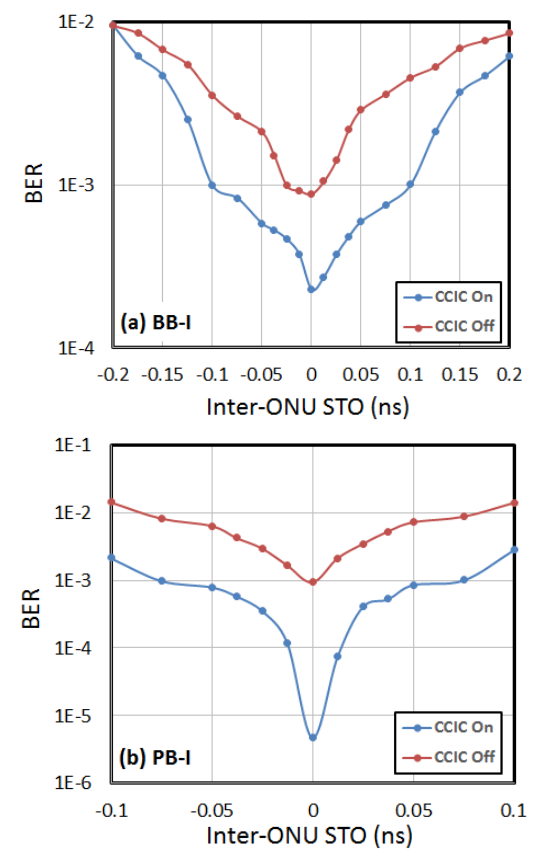

Fig. 7. Variation of BER with Inter-ONU STO for (a) BB and (b) PB in-phase channels with and without CCIC enabled

\section{Transparency of CCIC to Signal Modulation Format}

In this section, the transparency of the CCIC function to the interfering channel's modulation format is investigated. The DFMA PON, with all parameters as specified in Table I, is employed, with a total ROP of $-10 \mathrm{dBm}$. The ONUs are configured such that ONU1 (ONU2) generates the BB-I and PB-Q (BB-Q and PB-I) channels, thus the CCI-inducing orthogonal channels originate from different ONUs. The CCIC function is set for the previously determined optimum tap-count of 42. To evaluate the transparency to modulation format across the whole spectral region of a sub-band channel, all subcarriers are enabled and the BER versus subcarrier index is measured for PB-I and PB-Q for two different cases. For case 1, all channels adopt 16-QAM on all subcarriers and the system is optimised for minimum channel BERs using the procedure described in Section III-D. For case 2, the ONU generating the interfering orthogonal channel is configured to generate 32QAM on all subcarriers on both of its channels, whilst all other transmitter and receiver settings remain unchanged. The BER versus subcarrier index for both cases is plotted for ONU2 (16QAM on PB-I) and ONU1 (16-QAM on PB-Q) in Fig. 8(a) and Fig. 8(b) respectively. The results clearly show that the CCIC function is highly effective with either 16-QAM or 32-QAM modulation on the interfering orthogonal channel, showing that the CCIC function is highly flexible and transparent, as its operation is independent of modulation format, and the required CCIC function's configuration is also completely independent of the modulation format. Also, it can be seen in Fig. 8 that the shape of the BER versus subcarrier index curves are very similar for the two aforementioned cases, indicating the 16QAM and 32-QAM interfering signals generate a similar spectral distribution of interference signal power, this supposition is validated by the fact that the frequency spectra of the 16-QAM and 32-QAM interference signals are also very similar, as shown on Fig. 8(c) and Fig. 8(d) for the cases of
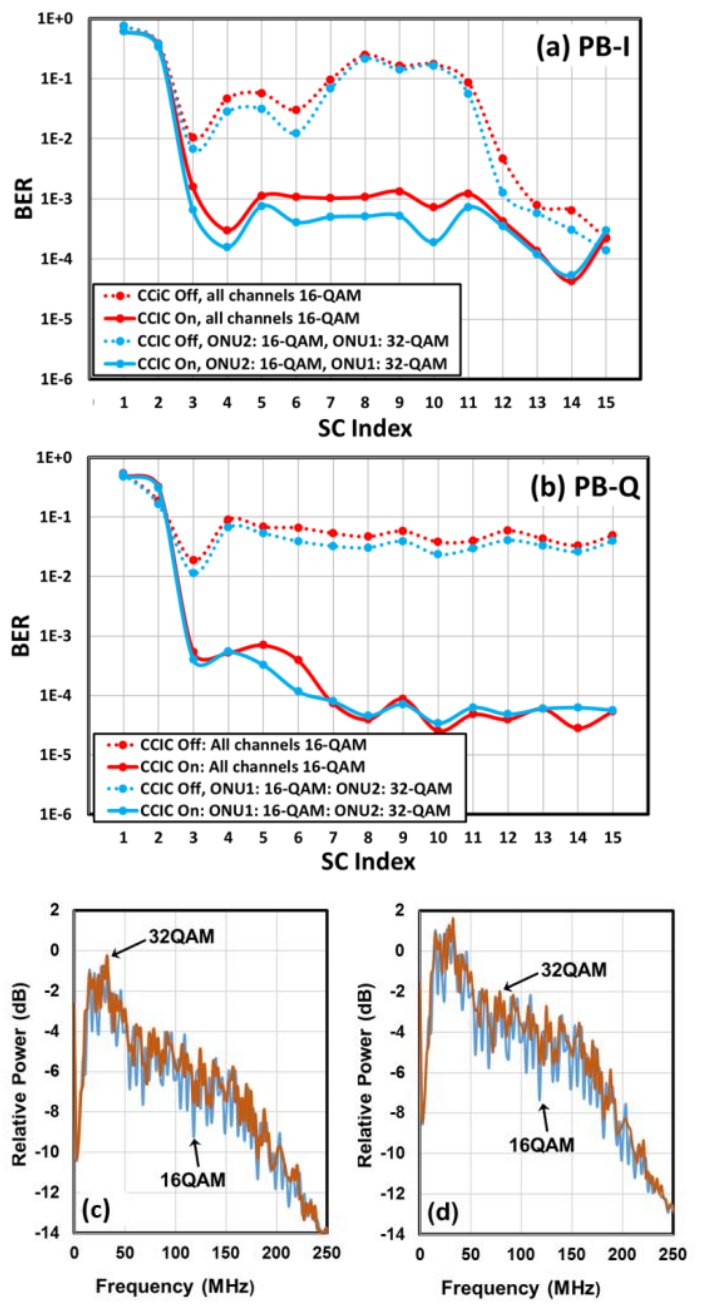

Fig. 8. BER vs. subcarrier index for $26 \mathrm{~km}$ SSMF multipoint-to-point transmission at an ROP of $-10 \mathrm{dBm}$ for (a) PB-I from ONU2, (b) PB-Q from ONU1 and corresponding interfering signal spectra (c) PB-I, (d) PB-Q

recovering the PB-I and PB-Q channels respectively. As the real-time receiver DSP is not designed to allow observation of the actual interference signal within the FPGA, the aforementioned spectra are generated using a Simulink-based simulation model of the experimental DFMA PON system, employing a digital FIR filter to represent the physical channel's response (DAC, E/O, fibre, O/E, ADC).

\section{DSP COMPLEXITY ANALYSIS}

\section{A. Complexity Optimisation of DSP-based CCIC filter}

In this sub-section, we examine the responses and the associated complexity of the CCIC filters for the 4 channel DFMA PON. Asumming the SFs and MFs are 64 taps in length and the determined channel response is 32 taps in length, the full length CCIC filters resulting from the convolution of the three concatenated filters would be 158 taps in length. Fig. 9 (ad) shows the CCIC filter impulse responses for the cases of receiving the BB-I, BB-Q, PB-I and PB-Q channels respectively. By inspection, it is easy to see that truncating the impulse responses to 64 taps would lead to minimal deviation from the full-length filter response, thus a tap count of 64 is 

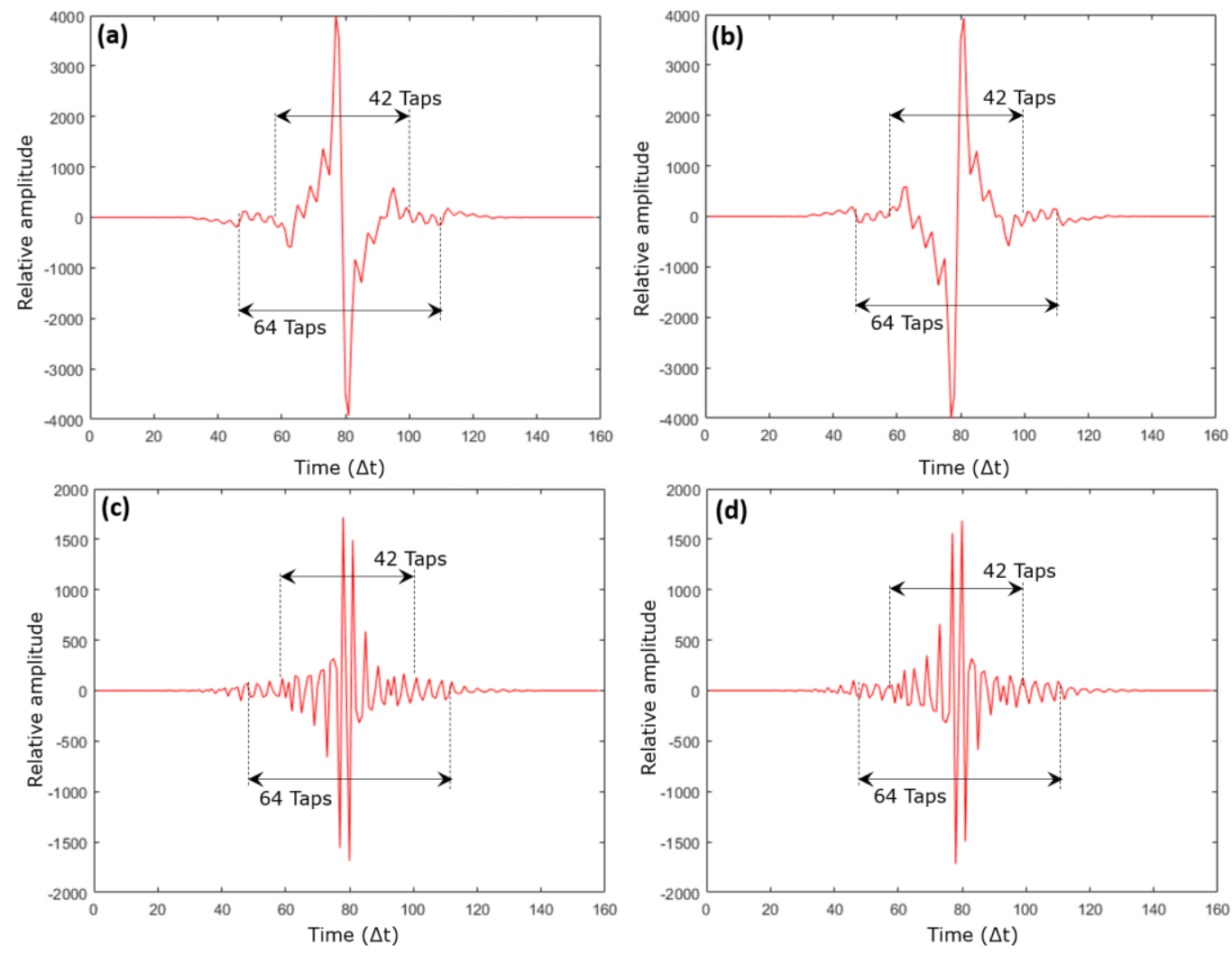

Fig. 9. Full length (158 tap) impulse responses of CCIC filters (a) BB-I, (b) BB-Q, (c) PB-I, (d) PB-Q

selected as the starting point for the CCIC filter tap count optimisation study.

In section III-C, it was concluded that 42 taps is a good trade-off between CCIC filter complexity and performance. To further validate this conclusion, Fig. 10 plots the BER values for each channel against tap count, extracted from the BER curves in Fig. 3, at a total ROP of -14dBm. Fig. 10 shows that as tap count decreases below 42 the BER sharply increases as the CCIC filter's impulse response rapidly deviates from its desired response, thus reducing the accuracy of the estimated interference signal. However, as tap count increases above 42 the relatively small weighting of the additional taps gives little improvement in the accuracy of the CCIC filter's impulse response, and consequently little improvement in the accuracy of the estimated interference signal. Hence, 42 taps provide an optimum CCIC filter size. The CCIC filter complexity in terms

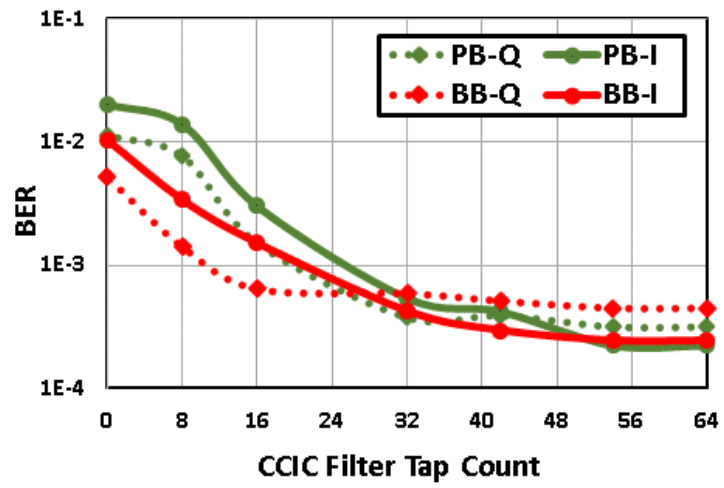

Fig. 10. BER vs. CCIC filter tap count for $26 \mathrm{~km}$ SSMF point-to-point transmission at ROP of $-14 \mathrm{dBm}$ of tap count (scalar multiplier count) is therefore reduced by $73 \%$ in comparison to the full-length CCIC filter.

\section{B. Complexity of Shaping, Matching and CCIC Filters in Multi-Channel DFMA PONs}

In this section the complexity of the SF, MF and CCIC filters, in terms of scalar multiplier count (tap count), is explored to determine the interdependency on the following parameters: channel count $N$, DAC/ADC sampling rate $S$ (proportional to total aggregate signal bandwidth) and maximum filter digital logic clock frequency, $K$. It should be noted that the required multiplier elements for all the digital filters are scalar, fixed-point multipliers and not the more logic resource hungry, complex, floating-point multipliers. Also, for the demonstrated DFMA PON incorporating CCIC, in the SF and MF (CCIC) filters, multipliers with only $8 \times 8$-bit (8x12-bit) resolution are employed.

The DSP architecture of the digital SF is shown in Fig. 11. Parallel FIR filters are employed, as typically the digital logic clock frequency is significantly lower than the ADC/DAC sample rate. As the sampling rate of the ADC/DAC is $S$ samples/s and the clock frequency of the digital logic is $K \mathrm{~Hz}$, $P$ can be considered as a parellisation factor and is defined as the ratio of $S$ to $K$, i.e. $P=S / K$, and so the required effective number of parallel filters is $P$. For $P$ parallel filters, $P$ parallel input samples are input to the filter bank and a delay function generates the necessary delayed samples to feed all FIR filters. To minimise the SF complexity in terms of the number of scalar multiplier elements required for the FIR filters, $P$ is selected to be an integer multiple of the up-sampling factor $M$. This ensures that the zero-valued samples, generated by the up-sampling 


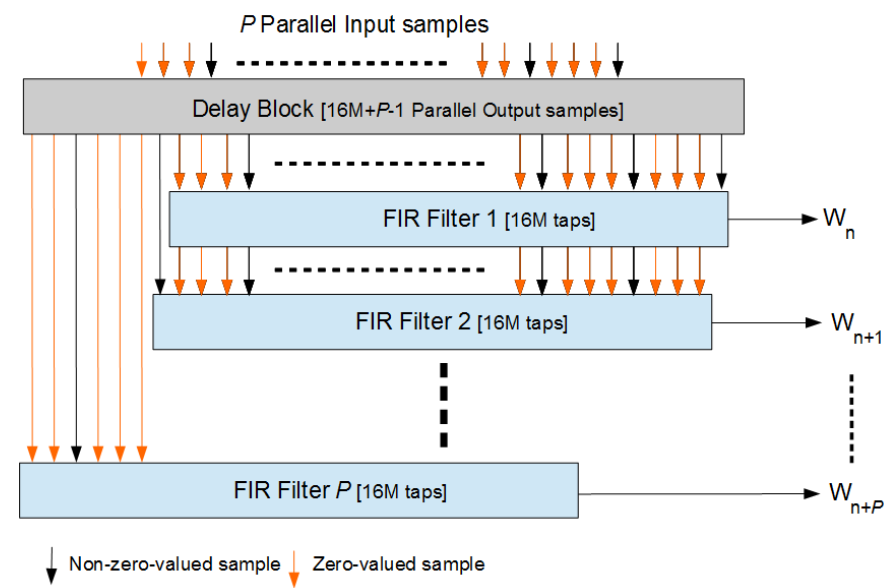

Fig. 11. Shaping Filter DSP Architecture

function, are always located at the same inputs on each FIR filter, this arrangement is therefore exploited to minimise the SF complexity. Fig. 11, illustrates the architecture for the case of $M=4$ as an example, however the structure is valid for the general case for all $M$ values, where every $M^{\text {th }}$ input sample is non-zero. In Fig. 11, the zero-valued (non-zero valued) samples are indicated by the orange (black) coloured arrows. In [4] an optimum length of the SF FIR filters was shown to be $16 \times M$, therefore each FIR filter always has 16 non-zero valued input samples irrespective of the up-sampling factor $M$. As the required number of parallel FIR filters, $P$, is $S / K$, the total number of scalar multipliers, $X_{S F}$, required for the $\mathrm{SF}$ is:

$$
X_{S F}=16(S / K)=16 P
$$

Note that as $P$ must be a multiple of $M$, the value of $K$ would be adjusted accordingly. As the maximum number of channels $N$, is equal to the up-sampling factor $M$ [4], this leads to the important result that the complexity of the SF is totally independent of $N$, thus scaling of the channel count does not lead to excessive SF complexity.

As the adopted sample rate is $2 \mathrm{GS} / \mathrm{s}$ and the SF clock rate is $125 \mathrm{MHz}, P=16$, therefore using Eq.(1), the number of scalar multipliers employed in the implemented SF described in section III-A, is $16 \times 16=256$. Also, as an example, a system operating at $50 \mathrm{GS} / \mathrm{s}$ and with a digital logic speed of $390.625 \mathrm{MHz}$ (i.e. $P=128$ ), would require $16 \times 128=2048$ scalar multipliers, which is entirely practical with modern high density application specific integrated circuit (ASIC) or FPGA technology [22].

The DSP architecture of the digital MF is shown in Fig. 12, where $P$ parallel FIR filters are again required to restrict the digital clock frequency to a practical value. The length of the FIR filters in terms of taps is again $16 \times M$ [4], however now there are no continuously zero-valued samples at the filter inputs so all FIR filters must employ $16 \times M$ scalar multipliers. If the filter bank operates on $P$ parallel input samples, a similar delay block as in the SF, is employed to generate all the appropriately delayed samples for the filter inputs. As there are $P$ parallel inputs to the MF there should be $P$ parallel outputs, however the subsequent down-sampling function discards $M-1$ samples in every $M$ samples, this fact can be exploited to completely eliminate the corresponding redundant FIR filters in the parallel filter bank, thus the total number of physically

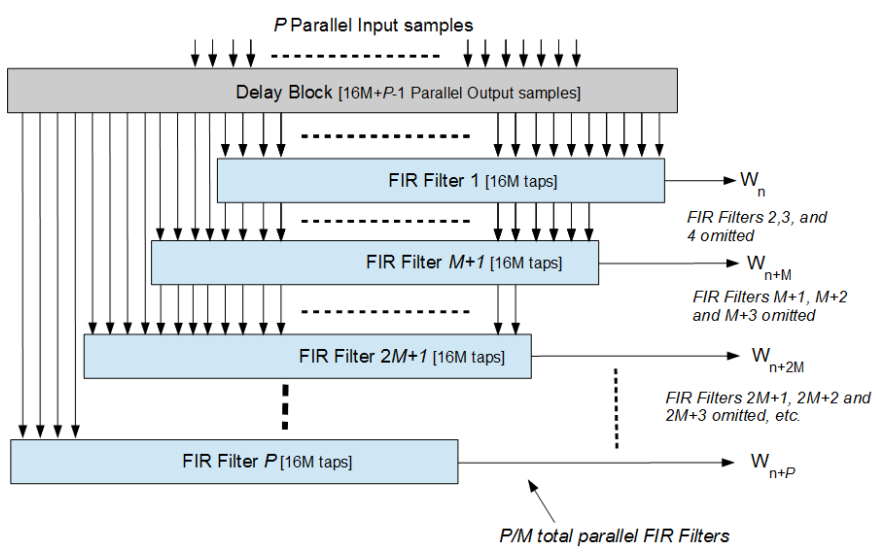

Fig. 12. Matching Filter DSP Architecture

implemented parallel filters is $P / M$, a reduction by a factor of $(M-1) / M$. It should be noted that $P$ must again be a multiple of $M$ to ensure the down-sampling discarded samples always originate from the same FIRs, and $P \geq M$ must be valid as there must be at least one FIR filter, corresponding to the case when $P=M$. As there are $P / M$ parallel FIR filters each with $16 \times M$ scalar multipliers, and assuming $P \geq M$, there are a total of $X_{M F}$ scalar multipliers in the MF, given by:

$$
X_{M F}=16 M(P / M)=16 P
$$

This is identical to the SF case and more importantly shows $X_{M F}$ is independent of the maximum channel count $\mathrm{N}$, so MF complexity also does not scale with channel count. The implemented MF described in Section III-A, thus also employs 256 scalar multipliers.

The DSP architecture of the CCIC filter is shown in Fig. 13, which is based on a combination of the SF and MF filter structures. As the CCIC filter is preceded by up-sampling and followed by down-sampling, it can exploit the multiplier redundancies present in both the SF and MF filter architectures, specifically, no multipliers are required for zero-valued input samples and no FIR filters are needed where the output sample is discarded in down-sampling. For the demonstrated 4 channel case, each filter supports a tap count of 42 , thus with $M=4$ the number of scalar multipliers per FIR is 11 . As $P=16$ and $M=4$ the number of parallel FIR filters is 4 , so there are only $11 \times 4=44$

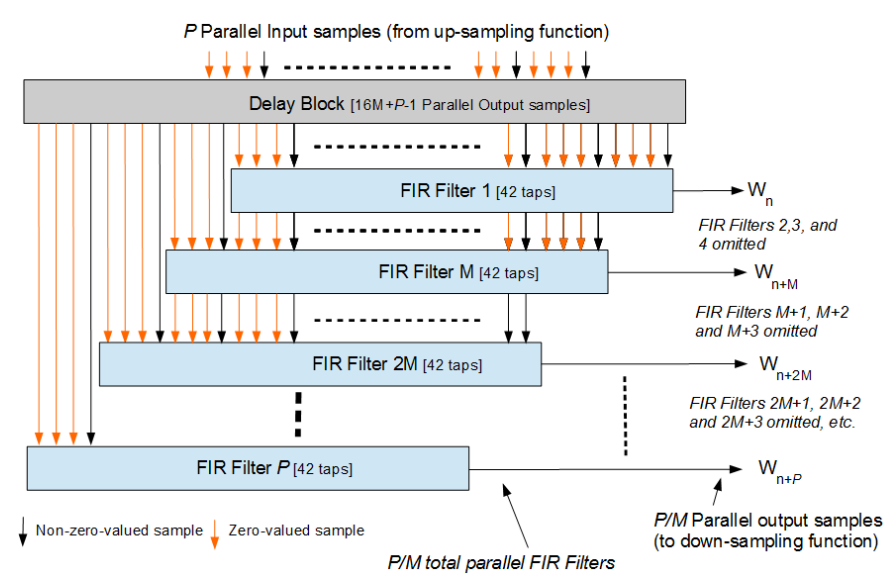

Fig. 13. CCIC Filter DSP Architecture 


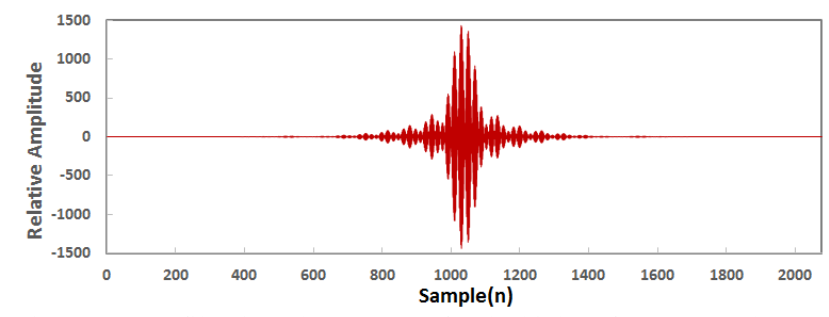

Fig. 14. CCIC filter impulse response for the highest frequency subwavelength band in a system with 64 channels, for the case of interference from I channel to Q channel.

scalar multipliers in the implemented CCIC function, thus an ultra-low complexity DSP design is achieved.

It is also important to consider how the complexity of the CCIC filter scales with parameters such as, channel count $N$ and the aforementioned $P$ value. To explore the possibility of truncating the CCIC filter impulse responses for DFMA PONs employing higher channel counts, Fig. 14 shows the CCIC filter impulse response for the case of 64 channels, CCI from I to Q on the sub-wavelength band with the highest central frequency and using the channel impulse response of the experimental system. It is clear that the resultant 2078-tap filter can be truncated to approximately $30 \%$ of the full length giving a 600-tap filter. CCIC filter responses for other tap counts and sub-wavelength bands are found to have a similar property.

The CCIC filter complexity in terms of total scalar multiplier count $X_{C C I C}$, can be determined for the general case, assuming tap count truncation to $30 \%$ of original count and adopting the DSP architecture of Fig. 13. Here we assume the channel count $\mathrm{N}$ is maximum such that $\mathrm{N}=\mathrm{M}$ [4]. The nominal length of the CCIC filter in taps will be $T_{S F}+T_{M F}+T_{C H}-2$, where $T_{S F}, T_{M F}, T_{C H}$ are the tap counts of the SF, MF and the estimated channel impulse response respectively. The nontruncated CCIC filter tap count is therefore $16 M+16 M+32-2=$ $32 M+30$, assuming $T_{C H}=32$. The truncated CCIC filter tap count is therefore:

$$
X_{T R}=\lceil 0.3(32 M+30)\rceil=\lceil 9.6 M+9\rceil
$$

where $[\mathrm{a}]$ denotes the minimum integer not smaller than real value a. The number of scalar multipliers per FIR filter is reduced by a factor of $1 / \mathrm{M}$, due to zero-valued sample insertion by the $\mathrm{M} \times$ up-sampling function, thus multiplier count per FIR is:

$$
X_{F I R}=\lceil\lceil 9.6 M+9\rceil / M\rceil=\lceil 9.6+(9 / M)\rceil
$$

The number of parallel FIR filters taking into account the redundancy due to $\mathrm{M} \times$ down-sampling, is $[\mathrm{P} / \mathrm{M}\rceil$ and therefore the total number of scalar multipliers is given by:

$$
X_{C C I C}=\lceil 9.6+(9 / M)\rceil\lceil P / M\rceil
$$

A highly significant result here is that as $\mathrm{M}$, and therefore channel count $\mathrm{N}$, increases $X_{C C I C}$ in fact decreases and approaches a fixed value of only 10 . Therefore, ultra-low CCIC filter complexity is achievable for high channel count DFMA PONs. This key result shows that for $\mathrm{M} \geq 4$ the CCIC filter complexity in terms of scalar multipliers is $\sim 10 \times$ [number of parallel FIRs]. The implemented CCIC filters comply with this fact as the optimum tap count of 42 results in 11 scalar multipliers per parallel FIR filter. Therefore, as each parallel FIR generates a separate output sample, only $\sim 10$ sequential,

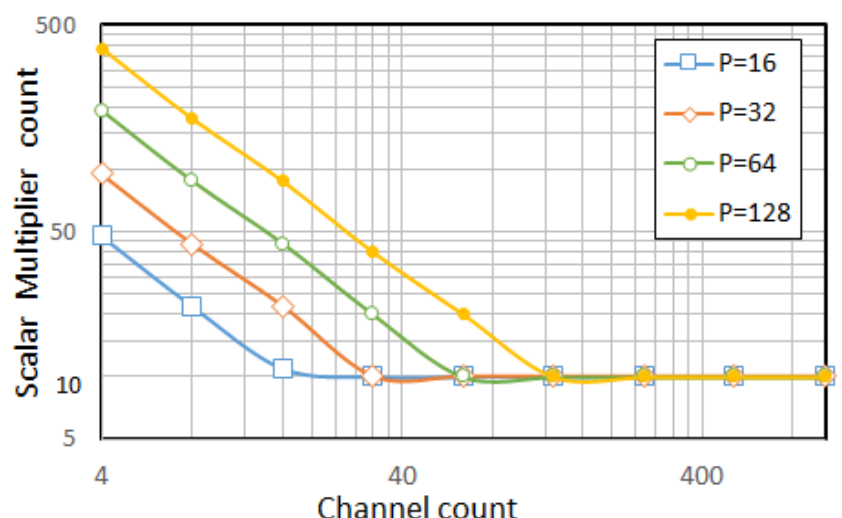

Fig. 15. CCIC filter scalar multiplier count $X_{C C I C}$, variation with channel count $\mathrm{N}$ (where $\mathrm{N}=\mathrm{M}$ ) for varying $\mathrm{P}$ values

non-zero valued samples from the CCI-inducing signal are required to produce each interference-estimate signal sample. This indicates that only 10 samples from the CCI-inducing signal generate significant interference in a single sample in the CCI-impacted signal. Fig. 15 illustrates the scalar multiplier count, $X_{C C I C}$, as defined by Eq. (5), for various channel counts up to 1024 channels, for four different $P$ values. It is clear that complexity decreases with channel count and as $M$ approaches $P$ the complexity $X_{C C I C}$ approaches a fixed value of only 10 multipliers.

It can therefore be concluded that the complexity of the DSP required for the ONUs and OLT in a DFMA PON does not scale with channel number, and as a channel is used by one or more ONUs, it does not scale with ONU count. The OLT requires DSP hardware per connected ONU, however using a modular hardware design would allow the aforementioned "pay-as-yougrow" PON deployment.

\section{CONCLUSION}

DFMA PONs can achieve SDN-controlled, physical layer network reconfigurability to offer highly efficient use of network resources, elastic bandwidth provisioning and fixed and mobile network convergence. To technically validate the DFMA PON technique a fully real-time upstream DFMA PON incorporating four independent OFDM modulated channels, has been experimentally demonstrated in a $26 \mathrm{~km}$ SSMF, IMDD-based PON consisting of two ONUs and an OLT, all of which are dynamically reconfigurable and operate completely in real-time. As physical channel frequency response-induced CCI severely degrades the performance of two spectrally overlaid orthogonal channels, the OLT receiver incorporates a low complexity real-time DSP-based CCIC function, which is shown to considerably improve the performance and robustness of the four ONU channels, this is evidenced by: i) individual OFDM subcarriers exhibit a reduction in BER by factors as large as $100-300$ times, ii) for the adopted FEC limit, the achieved channel capacities are significantly increased in terms of operational subcarrier count, for one channel 13(0) of 15 subcarriers can be employed when the CCIC is enabled (disabled), iii) sensitivity to ONU synchronisation is reduced as the inter-ONU STO range can be increased by a factor of up to 15 times, and iv) excellent transparency to the interfering channel's signal modulation format. 
The CCIC function is fully optimised for low DSP complexity and it has been shown that the associated CCIC filter requires as few as only $\sim 10$ scalar multipliers per parallel signal sample, which is independent of the implemented channel count. Furthermore, the DSP complexity analysis of the SFs and MFs, has also shown that their complexity in terms of scalar multiplier count is independent of channel count.

This work therefore demonstrates the technical feasibility of achieving high performance IMDD DFMA PONs incorporating DSP-based transceivers with low complexity digital orthogonal filters, and furthermore, that ultra-low complexity CCIC functions are highly effective in mitigating the CCI due to the non-flat physical channel responses.

\section{REFERENCES}

[1] Ciena, "White Paper: Introducing The Adaptive Network ${ }^{\mathrm{TM}}$ Vision," https://media.ciena.com/documents/Introducing-the-Adaptive-NetworkVision-WP.pdf, 2018.

[2] H. Chen et al., "Ultra-Reliable Low Latency Cellular Networks: Use Cases, Challenges and Approaches," IEEE Commun. Mag., vol. 56, no. 12, pp. 119-125, Dec. 2018.

[3] X. Costa-Pérez et al., "Network slicing for 5G networks," in $5 G$ Networks: Fundamental Requirements, Enabling Technologies, and Operations Management, Hoboken, NJ, USA: John Wiley \& Sons, Inc., 2018, pp. 327-370.

[4] M. Bolea, R. P. Giddings, M. Bouich, C. Aupetit-Berthelemot, and J. M. Tang, "Digital filter multiple access PONs with DSP-enabled software reconfigurability," J. Opt. Commun. Netw., vol. 7, no. 4, 2015.

[5] X. Duan, R. P. Giddings, S. Mansoor, and J. M. Tang, "Experimental demonstration of upstream transmission in digital filter multiple access pons with real-time reconfigurable optical network units," IEEE/OSA J. Opt. Commun. Netw., vol. 9, no. 1, pp. 45-52, 2017.

[6] P. Popovski, K. F. Trillingsgaard, O. Simeone, and G. Durisi, "5G Wireless Network Slicing for eMBB, URLLC, and mMTC: A Communication-Theoretic View," IEEE Access, vol. 6, pp. 5576555779, 2018.

[7] X. Duan, R. P. Giddings, S. Mansoor, and J. M. Tang, "Performance Tolerance of IMDD DFMA PONs to Channel Frequency Response Rolloff," IEEE Photonics Technol. Lett., 2017.

[8] E. Al-Rawachy, R. P. Giddings, and J. M. Tang, "Experimental demonstration of a DSP-based cross-channel interference cancellation technique for application in digital filter multiple access PONs," Opt. Express, vol. 25, no. 4, pp. 3850-3862, 2017.

[9] J. G. Andrews, "Interference cancellation for cellular systems: A contemporary overview," IEEE Wirel. Commun., vol. 12, no. 2, pp. 1929, 2005.

[10] N. I. Miridakis and D. D. Vergados, "A Survey on the Successive Interference Cancellation Performance for Single-Antenna and MultipleAntenna OFDM Systems," IEEE Commun. Surv. Tutorials, vol. 15, no. 1, pp. 312-335, 2013.

[11] L. Tao, Y. Wang, Y. Gao, A. P. T. Lau, N. Chi, and C. Lu, "40 Gb/s CAP32 System With DD-LMS Equalizer for Short Reach Optical Transmissions," IEEE Photonics Technol. Lett., vol. 25, no. 23, pp. 2346-2349, Dec. 2013.

[12] Y. Dong, E. Al-Rawachy, R. P. Giddings, W. Jin, D. Nesset, and J. M. Tang, "Multiple Channel Interference Cancellation of Digital Filter Multiple Access PONs," J. Light. Technol., vol. 35, no. 1, 2017.

[13] W. Shieh, "OFDM for Flexible High-Speed Optical Networks," J. Light. Technol., vol. 29, no. 10, pp. 1560-1577, May 2011.

[14] X. Duan et al., "Real-time experimental demonstrations of software reconfigurable optical OFDM transceivers utilizing DSP-based digital orthogonal filters for SDN PONs," Opt. Express, vol. 22, no. 16, 2014.

[15] R. P. Giddings, X. Q. Jin, E. Hugues-Salas, E. Giacoumidis, J. L. Wei, and J. M. Tang, "Experimental demonstration of a record high $11.25 \mathrm{~Gb} / \mathrm{s}$ real-time optical OFDM transceiver supporting $25 \mathrm{~km}$ SMF end-to-end transmission in simple IMDD systems," Opt. Express, vol. 18, no. 6, pp. $5541-5555,2010$.

[16] X. Q. Jin and J. M. Tang, "Experimental Investigations of Wavelength Spacing and Colorlessness of RSOA-Based ONUs in Real-Time Optical OFDMA PONs," J. Light. Technol., vol. 30, no. 16, pp. 2603-2609, Aug.
2012.

[17] A. Tsokanos et al., "Reductions of peak-to-average power ratio and optical beat interference in cost-effective OFDMA-PONs," Photonic Netw. Commun., vol. 26, no. 2-3, pp. 44-52, Dec. 2013.

[18] S.-M. Jung, S.-M. Yang, K.-H. Mun, and S.-K. Han, "Optical beat interference noise reduction by using out-of-band RF clipping tone signal in remotely fed OFDMA-PON link," Opt. Express, vol. 22, no. 15, p. 18246, Jul. 2014.

[19] Z. Li, M. S. Erkilinc, R. Bouziane, B. C. Thomsen, P. Bayvel, and R. I. Killey, "Simplified DSP-Based Signal-Signal Beat Interference Mitigation Technique for Direct Detection OFDM," J. Light. Technol., vol. 34, no. 3, pp. 866-872, Feb. 2016.

[20] J. Cho, C. Xie, and P. J. Winzer, "Analysis of soft-decision FEC on nonAWGN channels," Opt. Express, vol. 20, no. 7, p. 7915, Mar. 2012.

[21] A. J. Lowery, "Improving Sensitivity and Spectral Efficiency in DirectDetection Optical OFDM Systems," in OFC/NFOEC 2008 - 2008 Conference on Optical Fiber Communication/National Fiber Optic Engineers Conference, 2008, p. Paper OMM4.

[22] Intel, "Stratix 10 GX/SX Device Overview, Table. 4," https://www.intel.com/content/dam/www/programmable/us/en/pdfs/lite rature/hb/stratix-10/s10-overview.pdf, 2018. 